\title{
Distributed Data Acquisition System for Condition Monitoring of Low-speed Machines Using Wireless Sensor Networks
}

\author{
http://dx.doi.org/10.3991/ijoe.v12i01.5021
}

\author{
Kai Zheng, Yun Zhang, Lei Liu, Chen Zhao \\ Wuhan University of Technology, Wuhan, China
}

\begin{abstract}
Low-speed machines play an important role in industrial production, and the condition monitoring of these machines is of great importance. Monitoring with wireless sensor network (WSN) has many advantages. To monitor the condition of low-speed machines, we need to acquire low-frequency, weak and hardly-varying physical signals. As such, we designed a WSN system for high-precision signal acquisition. Actual measurement results showed that the acquisition precision of nodes could reach $0.01 \mathrm{mV}$. When the sensor nodes continuously acquired and sent data, the energy conversion efficiency was higher than $90 \%$ and the nodes' power consumption came to about $110 \mathrm{~mW}$. The WSN system was designed based on the low-power consumption 802.15.4 MAC/Zigbee, and the WSN was built through MESH topology. Data transmission was stable and the PER was lower than $1 \%$. The measurement results under laboratory and industrial field conditions showed that the WSN system designed met the requirements for on-site data acquisition and monitoring of low-speed machines.
\end{abstract}

Index Terms-Low-speed machines, Wireless sensor networks, High-precision signal acquisition, On-site data acquisition

\section{INTRODUCTION}

With the steady economic development in China in recent years, industry is playing an important role in economic production and has become a pillar industry in China's economy. Trouble-free operation of industrial equipment is crucial to guaranteeing industrial production. In particular, low-speed machines such as paper making machines, rotary drying drums, low-speed agitators and cement rotary kilns have been widely used in industrial production $[1,2]$. It is of great importance to monitor the condition and maintain the low-speed machines. Distributed data acquisition is a common method for condition diagnosis of industrial equipment.

Low-speed machines generally refer to the equipment with a rotating speed of less than $600 \mathrm{rpm}$. To monitor the condition of low-speed machines, according to [1, 2, 3, 4], we need to acquire low-frequency, weak-amplitude and hardly-varying physical signals (such as displacement, acceleration, tilt angle and deformation). These lowfrequency and weak signals are easily buried in external noise. To acquire these data, we need to take into account the sensors and data acquisition system. It is very important to select appropriate sensors, but it is not what this paper focuses on. When designing the data acquisition and monitoring system, we need to consider the following factors: (1) The data acquisition system does not need to have high-precision acquisition frequency as the signals to be acquired are often in low-frequency. (2) The data acquisition system should have high resolution and acquisition precision as the signals to be acquired are often weak ones. (3)The noise disturbance should be avoided during the signal transmission.

There are many problems with the wired method for monitoring the condition or acquiring the physical parameters of large industrial equipment, that is, complex wiring, high installation and maintenance costs $[5,6,7,8]$, easily aging cables due to the environment, signal transmission attenuation and noise disturbance $[5,6]$. Furthermore, it is impossible to acquire data with wired method on some special occasions such as when acquiring the surface temperature of a rotating shaft.

Using WSN for condition monitoring of industrial equipment has great application prospect. Compared with traditional wired method, WSN-based monitoring features low installation cost and flexible monitoring (it can realize short or long-time monitoring) and has no noise disturbance and signal attenuation resulting from the cables. Through this monitoring method, the measurement points can be flexibly deployed according to the measurement needs and the system can be easily expanded. Moreover, WSN is also the best solution for temporary installation when troubleshooting or testing machines [6]. Therefore, WSN has gained wide attention in the field of research on condition monitoring of industrial equipment.

According to the requirements for condition monitoring of low-speed machines, we designed a WSN system prototype for on-site data acquisition, condition monitoring and testing of low-speed machines. Our goal is to realize a WSN system with the following features:

- The nodes support continuous data acquisition and can work continuously for more than 20 days given battery power.

- The nodes have high acquisition resolution of analog quantities and support the access of various high-and low-frequency sensors so as to realize high-precision distributed data acquisition.

- he network has the function of automatic configuration and can realize self-organization of network under different industrial conditions and stable and reliable data transmission in industrial fields.

- he software system can realize parameter configuration, for example, selecting the sampling frequency of the wireless sensor nodes and sensor parameter calibration, and can manage, analyze and process the data acquired. 
Our discussion for wireless communication protocol is based on IEEE802.15.4 which features low complexity, self-organization, low power consumption and low cost $[14,15,16]$.

The remainder of this paper is organized as follows: In section 2, a review of the state-of-the art and the investigation of WSN for industrial equipment measurement and condition monitoring are presented. Section 3 presents the detailed implementation of the developed system. In section 4, we did experiments to evaluate the performance of the wireless sensor network system in the laboratory and in industry field. And conclusions are drawn in section 5.

\section{RELATED WORKS}

A wide range of researches have been conducted on the condition monitoring of low-speed machines. In this respect, the researches usually focus on: (1) designing a system for data acquisition and condition monitoring of low-speed industrial equipment; and (2) judging the condition of the low-speed equipment based on measurement principle and signal processing. Due to limited space, this paper focuses the discussion on the first part, that is, design of a distributed data acquisition system for condition monitoring of low-speed machines. In this section, we discussed and reviewed the hardware and software design and monitoring method of the data acquisition system for the industrial equipment.

In literature [1], the researchers designed a PLC-based system for data acquisition and monitoring of low-speed cement rotary kilns. This wired system is suitable for long-term equipment monitoring. In literature [4], the authors adopted AE measurement method to monitor lowspeed rotary equipment and designed a data acquisition system (with a low resolution) based on eight-bit data acquisition card and Matlab. As described in section 1, the wired method for data acquisition has many shortcomings. In literature [9], the authors used commercial wireless sensor to monitor the rotating equipment in nuclear reactors, and pointed out that wireless sensors were easy to be deployed, contributed to efficiency and economy, and was suitable for monitoring large rotating equipment. Also, they established the requirements and procedures to be followed when implementing wireless sensors for predictive maintenance in research reactors. This system was mainly targeted at general rotating machines. In literature [10], the authors designed a wireless sensor platform to collect the quantity of vibration for mechanical condition based monitoring (CBM -WSN). The hardware platform utilized the FPGA controller and an external 16-bit AD chip. The system was mainly designed for high-speed machines. However, the power consumption of the node was high and couldn't work long time if the node was powered with batteries. In literature [11], the authors designed a wireless sensor network based on Zigbee protocol and LabVIEW-2012 platform for industrial real-time measurement and monitoring system, and also discussed statistics and analysis of measurement data. There was no discussion related to designing a low power consumption sensor node or problem of high-precision acquisition of physical parameters. In literature [12], the authors proposed a wireless network system for condition monitoring in industrial systems. This platform implemented was customized node including PIC microcontroller, nRF2401A radio transceiver and an internal 10-bit analogto-digital conversion chip (ADC). And they used Dynamic
Power Management with Scheduled Switching Mode (DPM-SSM) protocol to extend the node lifetime. However, this system had low data acquisition precision, and it is unsuitable to use them to acquire weak signals.

\section{SYSTEM DESCRIPTIONS}

\section{A. Network architecture}

The framework diagram of the WSN designed for data acquisition and condition monitoring of the low-speed industrial equipment is shown in Figure 1. It mainly included the following parts: (1) Sensing layer, which mainly comprised the front-end sensors and the wireless sensor nodes on the equipment. The nodes were used to acquire the data from the sensors. The data transmission network between nodes was built through MESH topology. (2) Network layer, which included the base station and central monitoring station. Specifically, the base station received the data from the nodes and sent them to the central monitoring station. The software system in the central monitoring station could realize parameter configuration, data storage and analysis, condition monitoring and diagnosis, etc. (3) Application layer, which consisted of terminal computer and server and realized data transmission and sharing within the wide area network. (4) Moreover, the designed WSN system supported bidirectional communication between the users and the sensor nodes. All the data collected by the sensor nodes was sent to the upper computer while users can issue commands to the sensor nodes.

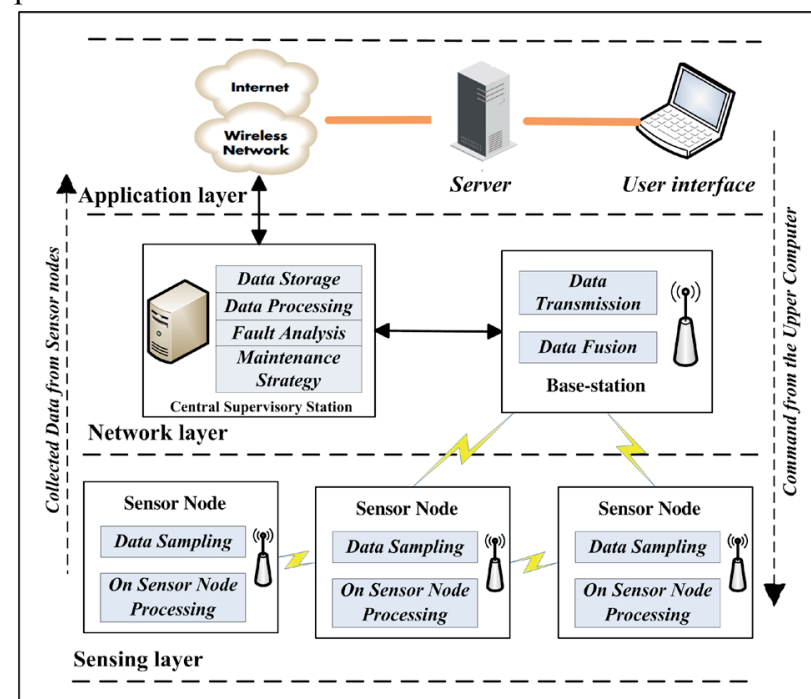

Figure 1. Architecture of WSN for condition based monitoring system

\section{B. Hardware implementations of sensor node}

The designed sensor node for high-precision data acquisition in this system is suitable for acquisition of lowamplitude and low-frequency analog signals. The system diagram and physical map of the node are shown in Fig. 2(a) and Fig. 2(b) respectively. The node comprised power supply module, communication module, processing unit and sensor module. Following is a detailed description about each module.

Power supply module. There are two common power conversion modes: Low drop output model (LDO) and DC-DC model. The conversion of LDO converter is usually accompanied with low noise and high power supply rejection ratio (PSRR). However, the input voltage should 

USING WIRELESS SENSOR NETWORKS

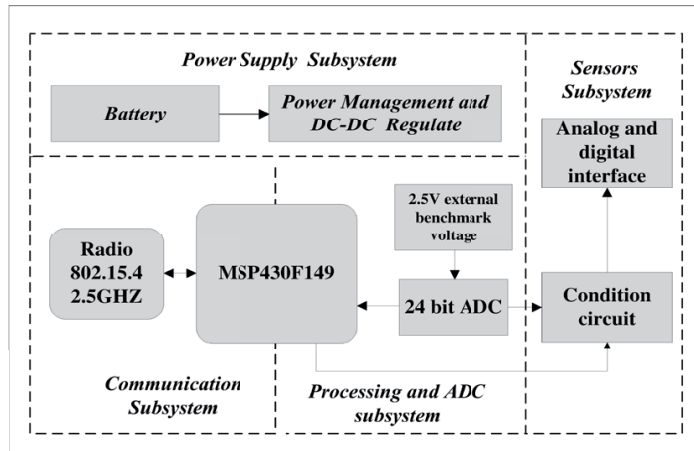

(a)

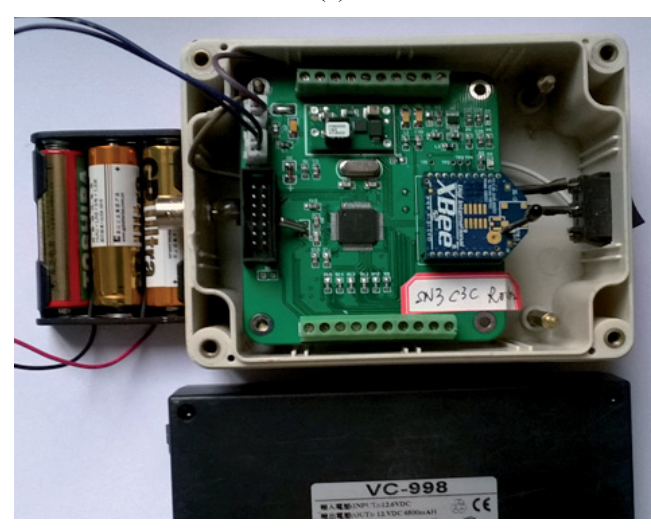

(b)

Figure 2. (a) Architecture of the sensor node (b) Prototype of the sensor node

be higher than the output voltage. Moreover, its conversion efficiency is low (generally lower than 80\%). Although DC-DC converter can realize high-efficiency conversion and is suitable for battery-powered equipment, the ripple of its output voltage is larger.

To maximize power conversion efficiency, we used DC-DC voltage conversion method to provide benchmark voltage for various modules. In design, power management IC TPS62740 and PTN0405C were used, whose conversion efficiency could reach over 90\% under PSM (Power Save Mode) and PWM (Pulse Width Modulation) modes. In the meantime, the output voltage of the power supply was filtered by second-order filter circuit (RC $\pi$ type filter) to attenuate the ripple produced during DC-DC conversion, with the output voltage precision reaching $0.5 \%$, thereby providing stable benchmark voltage.

Processing and sensing module. In design of the signal processing and communication main-board, MSP430F149 was used as the main control chip to acquire data as it is featured with low-power consumption.

To realize high-precision acquisition of weak signal in low speed machines such as vibration and displacement, an external low-power consumption 24-bit ADS1256 chip (power consumption $<38 \mathrm{~mW}$ under working mode) and $2.5 \mathrm{~V}$ external precision reference voltage source ADR421 were adopted. With a built-in PGA, ADS1256 is suitable for high-precision data acquisition as the amplification coefficient can be set according to measurement conditions. And it is powered by $5 \mathrm{~V}$ analog power supply and exchanges data with MSP430F149 through SPI protocol. Given full range and offset correction, the transfer function of ADS1256 is shown in formula (1)

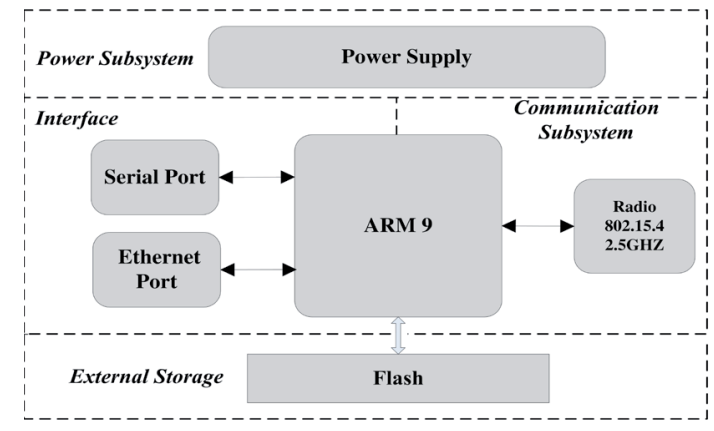

(a)

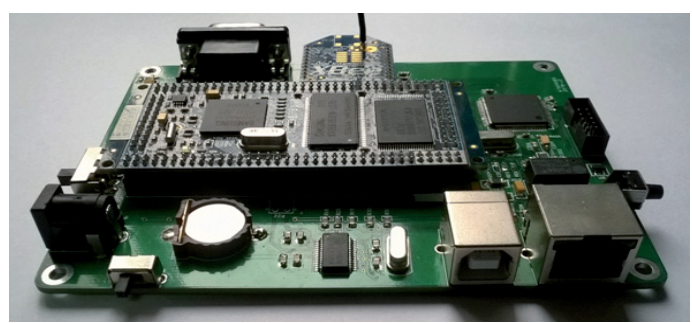

(b)

Figure 3. (a) Architecture of the based station (b) Prototype of the based station

$$
\text { Output }=\left(\frac{P G A \bullet V_{I N}}{2 V_{R E F}}-\frac{O F C}{\alpha}\right) F S C \bullet \beta
$$

Where Output is a 24-bit binary output code, $V_{I N}$ is the input voltage, $V_{R E F}$ is the external reference voltage, and $a$ and $b$ are constants, which change with the output speed of data and are set out in the table. OFC (Offset Calibration) is a complement code ranging from $-8,388608$ to $8,388,607$, and FSC (Full-Scale Calibration) ranges from 0 to $16,777,215$.

Communication unit. The XBee module was chosen as it supports Standard IEEE 802.15.4 and has ZigBee protocol stack. Furthermore, data transmission between ZigBee and MSP430 is achieved via UART protocol while featured with low-cost and low power consumption and long-range communication.

\section{Base station}

The base station plays an important role in the WSNCBM architecture since it coordinates the communication activity among the wireless nodes and serves as a gateway between the local system and the upper computer. According to IEEE802.15.4/Zigbee protocol, the wireless module of the base station nodes should be configured as a coordinator. The base station (as shown in Fig.3) is composed of power supply module; computer interface module (RS232 and Ethernet port); main control unit (ARM9-TQ2440) and wireless communication module, receiving the data collected by WSN nodes and sending them to the computer. All the data are saved by MySQL database in the computer. The database stores the relevant characteristics of the system nodes (Node ID, Sensor data, time, etc.) 


\section{NETWORK MANAGEMENT}

\section{A. Wireless protocols}

In the system, the network between the sensor nodes was built through ZigBee protocol. In IEEE802.15.4/Zigbee protocol, network topology can be classified as star topology, tree topology and MESH topology [14]. In particular, AODV routing protocol is adopted for MESH topology. This routing protocol is an on-demand routing algorithm based on distance vector, that is, it only maintains the necessary routing and does not need to maintain the routing that does not reach the destination node during the communication course. The nodes only need to remember the next routing path instead of the whole path. This protocol can set up a routing dynamically and automatically between various mobile nodes in the network. It can realize communication through multi-routing and automatically establish network communication. Its network topology is complicated but the transmission of network data is stable. In industrial field measurement and monitoring, the working conditions are usually harsh and there is electromagnetic interference. To realize stable work of sensor network, the MESH network topology is adopted in this system. In this research, the MESH network topology was configured by the X-CUTE software, and all the sensors node was set to routing node, as shown in Fig.4 (a). After the configuration was completed, the MESH network topology is shown in Fig. 4(b).

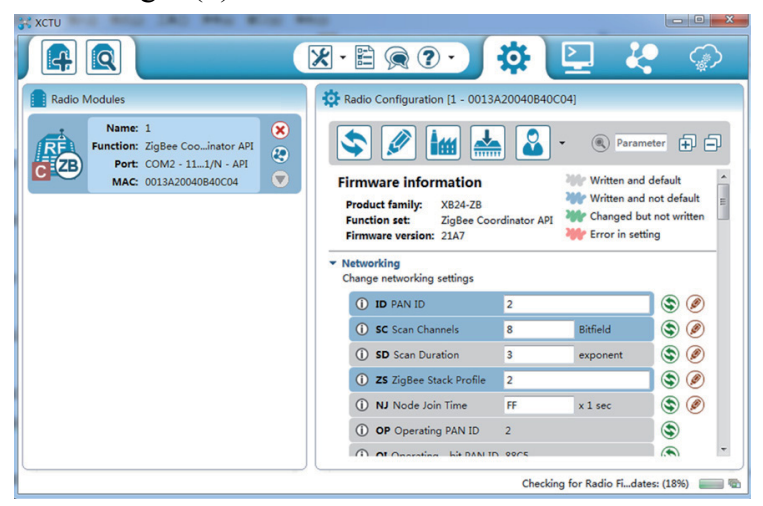

(a)

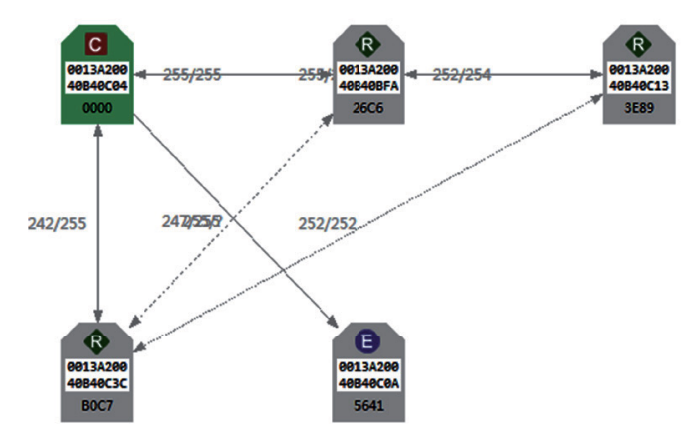

(b)

Figure 4. (a) X-CUTE software configuration (b) MESH network

\section{B. Software implementation}

This program was developed under the IAR C language in Integrated Development platform. We trans- planted the $\mu c-O S$ operating system to the nodes. The data acquisition algorithm consisted of four task modules, namely, AD data acquisition module, external crystal management module, wireless data sending and receiving module, and data checking module. Below is the algorithm process. First, the modules were initialized, including AD module, crystal module and wireless data configuration module. After the system was successfully initialized, external AD data acquisition started. Then, the acquired data were sent out by the wireless module.

The software in this system was developed based on Labview virtual instrument [17]. It was developed by adopting the multi-loop state machines based on producerconsumer queue. There were mainly four loops comprising event-driven loop, function processing loop, data acquisition loop and data error processing loop. By fully leveraging the multithreading operation of the

Labview-2012 development platform, the loops did not interfere in each other. The software interface is shown in Fig. 5. The software had the following functions:

- According to API protocol (as showed in Fig. 6), it could analyze the data packets received (as shown in Table II) from each sensor node and store the data into MY-SQL database.

- It could remotely configure the work mode of the nodes and automatically calibrate the sensors. Moreover, it could display the working condition of each node in real time, and automatically judge which node has been involved in a fault.

- It could process the data acquired online and offline, so as to diagnose the operating condition of the equipment and provide early warning for the equipment with the acquired data.

TABLE I.

PACKET OF THE SENSOR NODE

Received Packet:

7E0012900013A20040B40C133E8901010108080700C6

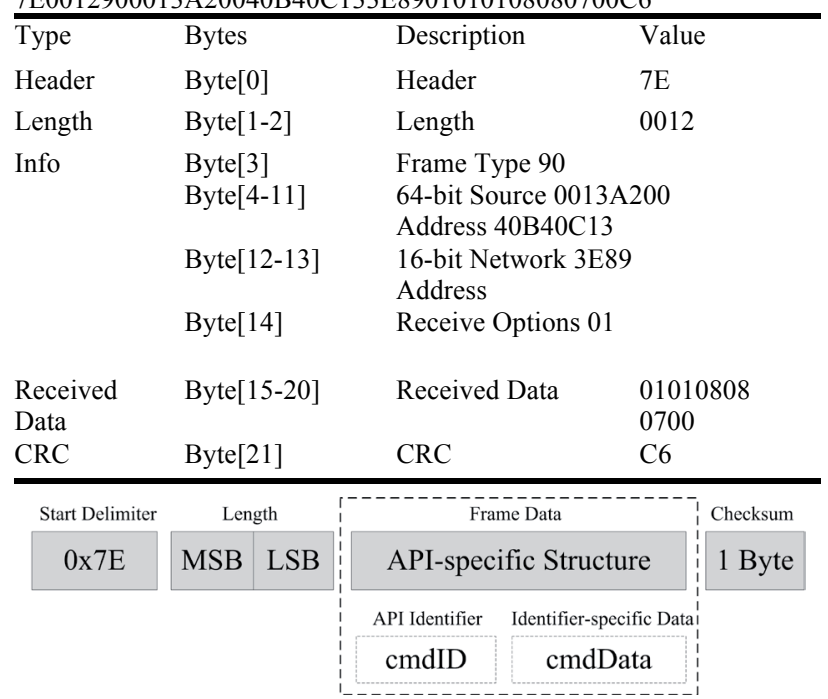

Figure 5. Wireless node communication data frame 


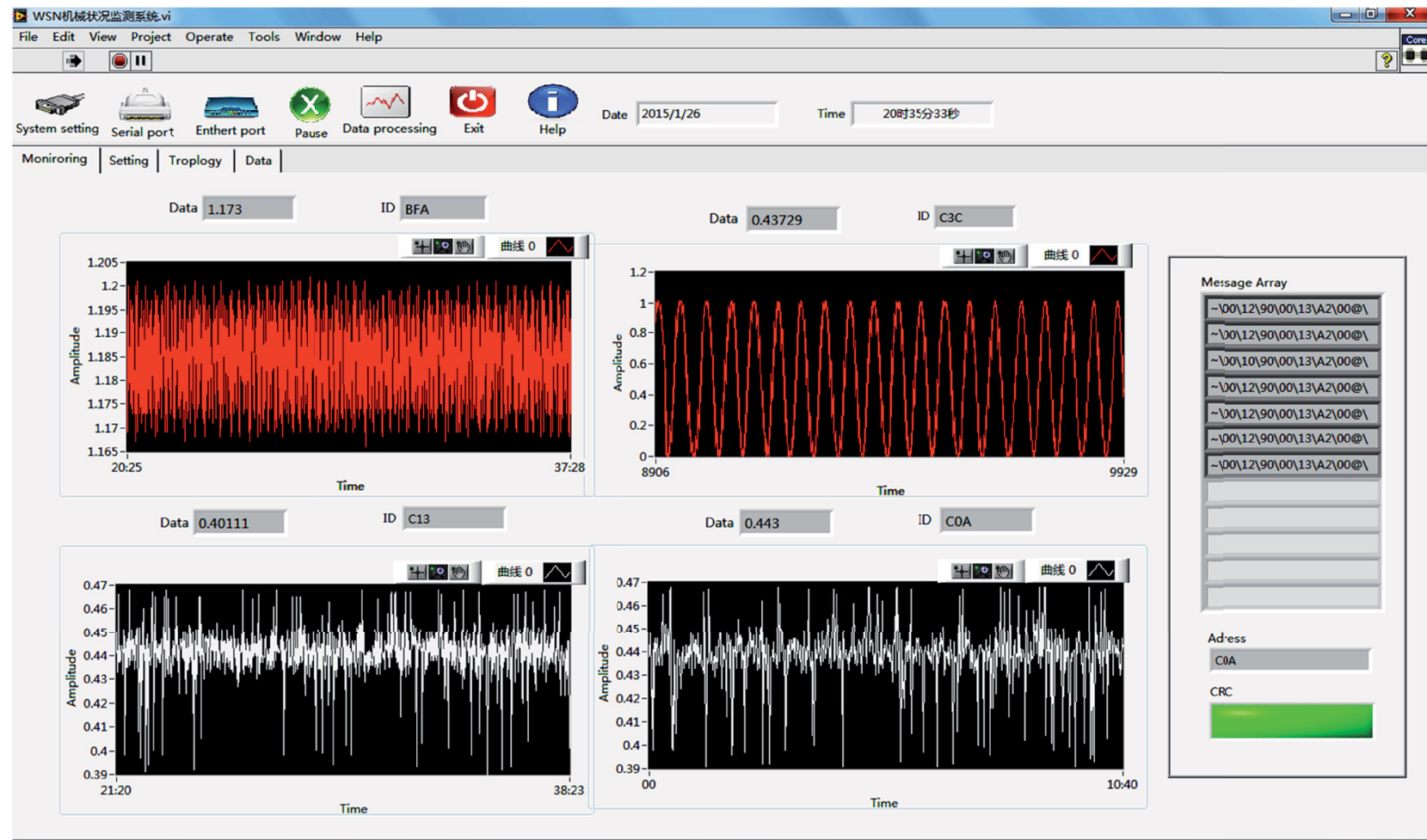

Figure 6. Front panel of the software for wireless data acquisition

\section{EXPERIMENTAL RESULTS}

\section{A. Laboratory validation}

We designed an experiment to verify the sampling precision of the system, as shown in Fig. 7. First, the output signals from Channel 1 of the signal generator were set as sinusoidal signals with amplitude of $4 \mathrm{mV}$ and frequency of $0.5 \mathrm{HZ}$. These signals were used to simulate weak signals. And the output signals from channel 2 were set as sinusoidal signals with amplitude of $5 \mathrm{mV}$ and frequency of $0.5 \mathrm{HZ}$. Meanwhile, the amplitude of this signal was adjusted from $5 \mathrm{mV}$ to $6 \mathrm{mV}$ to simulate the dynamic changes of weak signal. The signal input end of Node 1 was connected to Channel 1 of the signal generator and the signal acquisition end of Node 2 was connected to Channel 2. The results of the time domain and frequency domain of the signals acquired are shown in Fig. 8. It can be seen from Fig. 8 that the nodes designed can acquire integral weak input signals. Meanwhile, the nodes can achieve an acquisition precision of $0.01 \mathrm{mV}$. The actual resolution of $\mathrm{AD}$ conversion signals can reach 20 bits. It shows that the nodes designed support high-precision data acquisition. So, they can meet the requirements for data acquisition of low-speed machines.

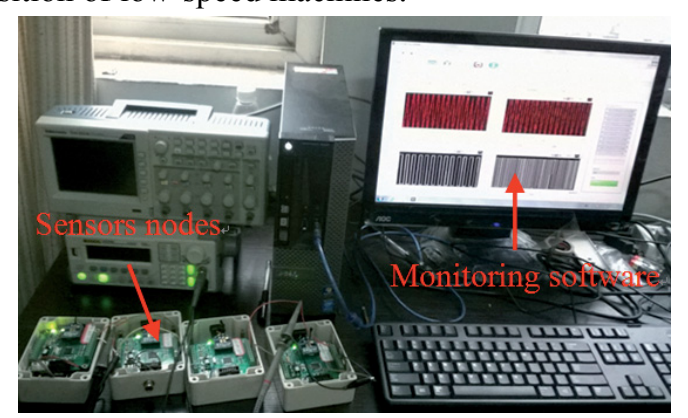

Figure 7. Physical diagram of the experiment
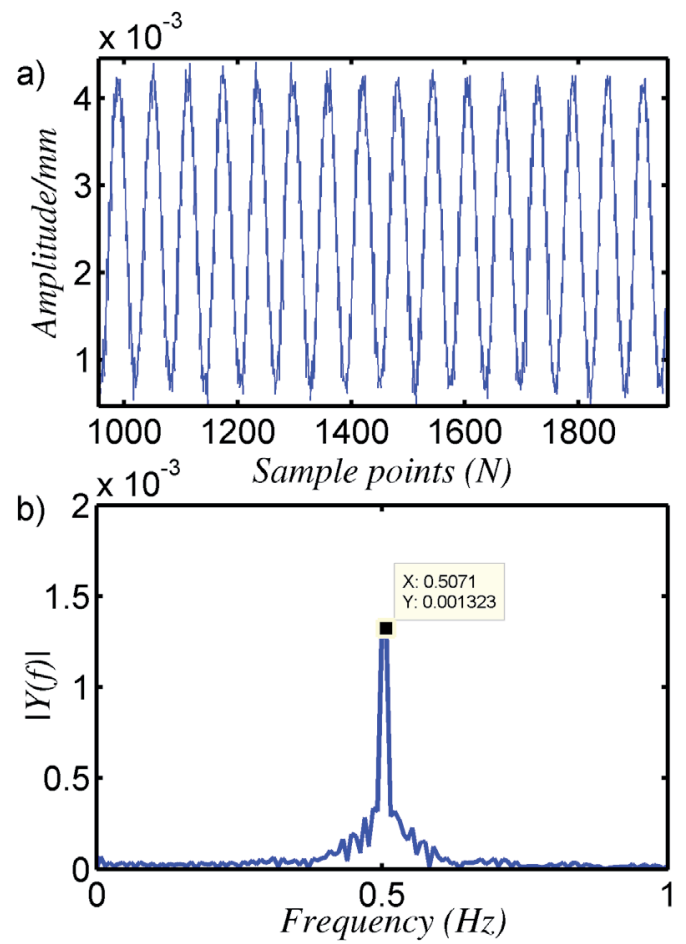

Figure 8. Results of time domain and frequency domain of the weak signals acquired by Node 1

TABLE II.

CURRENT CONSUMPTION OF THE MODULE

\begin{tabular}{cc}
\hline Type & Parameters (approximations) \\
\hline MSP430 & Working mode and time: $300 \mathrm{uA}, 1 \mathrm{~ms}$ \\
ADS1256 & Working mode and time: $7.45 \mathrm{~mA}, 2 \mathrm{~ms}$ \\
XBee & Working mode and time $: 45 \mathrm{~mA}, 10.9 \mathrm{~ms}$ \\
& Cycle of working time: $14.3 \mathrm{~ms}$ \\
\hline
\end{tabular}




\section{DISTRIBUTED DATA ACQUISITION SYSTEM FOR CONDITION MONITORING OF LOW-SPEED MACHINES USING WIRELESS SENSOR NETWORKS}

\section{B. Packet error rate}

To measure the stability of the sensor network, we placed four nodes and one base station node in the laboratory. In particular, three nodes were configured as routing nodes and one was configured as terminal node. A MESH network was built between nodes. Moreover, the nodes were set to work continuously with a data sampling frequency of $100 \mathrm{HZ}$ and sending rate of 9,600 bps. The PER of the data transmission of the two nodes upon actual measurement is shown in Fig. 9. It can be seen from the figure that the Packet error rate (PER) upon actual measurement is lower than $1 \%$ with the change of time.

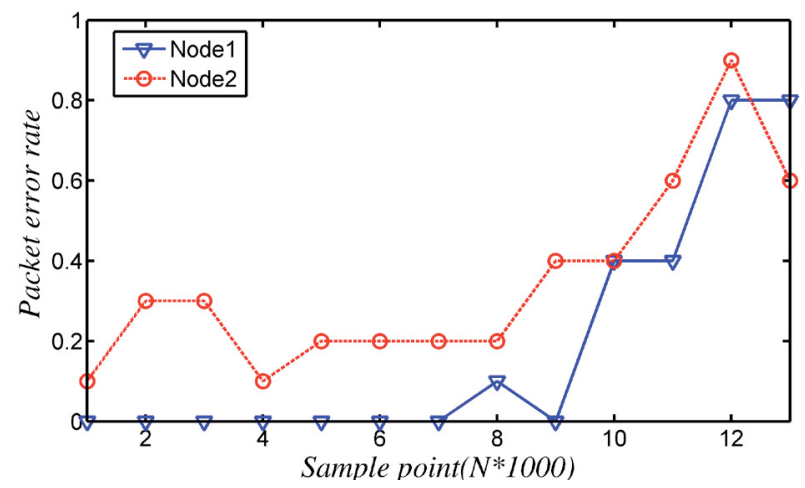

Figure 9. Packet error rate of the nodes

\section{Power consumption and life estimation of the sensor node}

There is a positive correlation between the WSN system's working time and the nodes' life. The working time of the battery-powered nodes depends on the battery life. That is, the nodes' life can be predicted by monitoring the battery life. Supposing the relationship between the nodes' working time and the supply voltage, current and resistance is expressed as $T=f(V, I, R)$, the average current consumed by the nodes working continuously can be expressed in formula (2). Specifically, $I_{R X}$ is the wireless emission current, $I_{A}$ is the working current of ADC chip, $I_{c}$ is the current consumed by MSP430, with the specific parameters set out in Table 2 .

$$
I_{\text {mean }}=\frac{T_{R X}}{T} I_{R X}+\frac{T_{A}}{T} I_{A}+\frac{T_{c}}{T} I_{c}
$$

According to the parameters in Table. 2 and formula 2, the current consumed by the nodes is about $35.6 \mathrm{~mA}$. Then, the power consumed by the nodes working continuously is calculated as

$$
W_{\text {mean }}=V_{\text {mean }} \times I_{\text {mean }}=117.4 \mathrm{~mW}
$$

The estimated working time of the nodes powered by $4.5 \times 600 m A h$ alkaline

is $(4.5 \mathrm{~V} \times 600 \mathrm{mAh}) / 117.4 \mathrm{~mW}=25 \mathrm{~h}$. When the WSN system was configured as MESH network, the nodes' data sending rate was $9600 \mathrm{bps}$ and the nodes were powered by $4.5 \times 600 \mathrm{mAh}$ alkaline battery, we measured the change trend of the three nodes' voltage (including two nodes configured as routers and one configured as terminal node), as shown in Fig. 10. The measurement result showed that the actual working time of the terminal nodes was longer than 30 hours. It indicated that the power actu- ally consumed by the terminal node was lower than 110 $\mathrm{mW}$ and that consumed by the routing nodes was about $118 \mathrm{~mW}$. If the node was powered by $16000 \mathrm{mAH}^{*} 5 \mathrm{~V}$ high-capacity lithium battery in actual measurement, the nodes could work continuously (continuously acquire and send data) for about 727 hours. So, they can meet the requirements for data acquisition and monitoring in industrial fields.

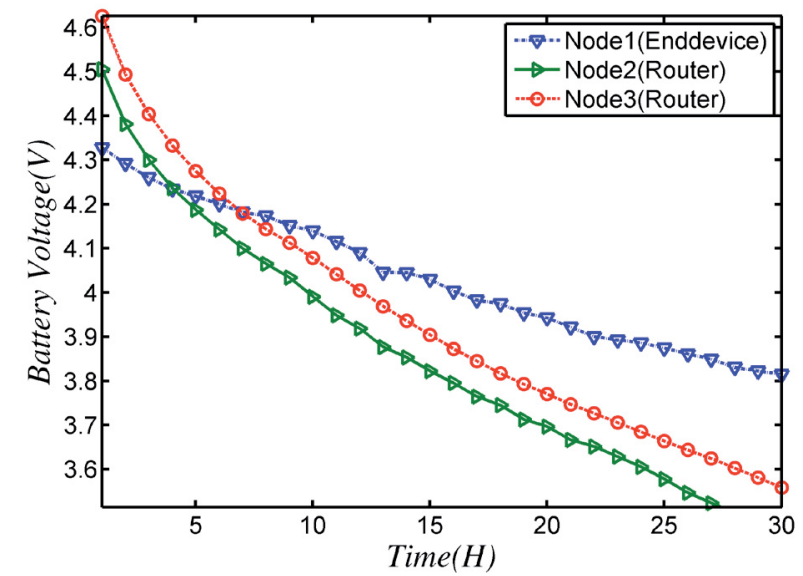

Figure 10 . Trend of the battery voltage for the nodes working continuously

\section{System test in industry field}

We validated the designed WSN data acquisition system in a real industry scenario. And the monitoring object is rotary kiln which is a critical equipment of metallurgy industry, cement industry and chemical industry. The rotary kiln is comprised of a kiln cylinder, support rollers and supporting bases. And it is a typical low speed machine as the operating speed of kiln cylinder is about 4rpm while the speed of support rollers is about 20rpm. As shown in Fig.11, two sensor nodes were placed on support roller\#1(upper roller) and support roller\#2(middle roller) separately and a base station was connected to the computer with RS-232 Port. In particular, the obliquity sensors connected to the nodes were used to acquire the tilt that supports the rollers in the horizontal direction. Where $I$ is the output current of the obliquity sensor, and $\theta$ is the tilt after conversion. In Fig. 12 about the results of actual data acquisition, the main vibration frequencies of the rollers were $0.06 \mathrm{HZ}$ and $0.36 \mathrm{HZ}$. The actual acquisition results showed that the nodes we designed could acquire weak physical signals.

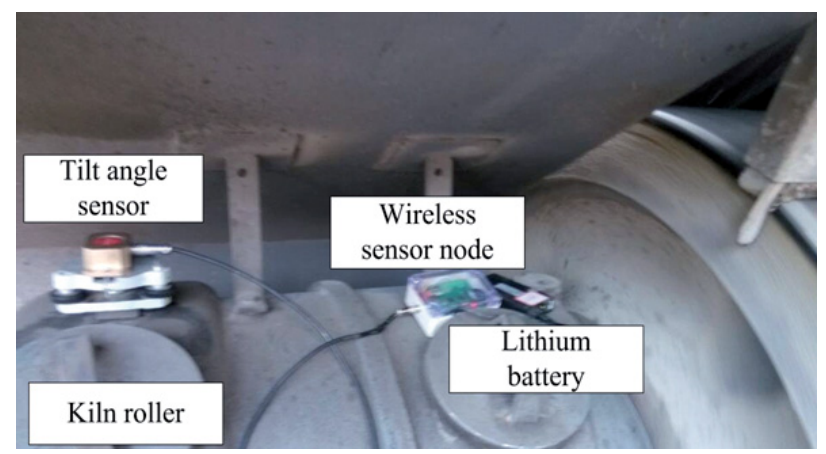

Figure 11. Wireless sensor system installed in the industry field 

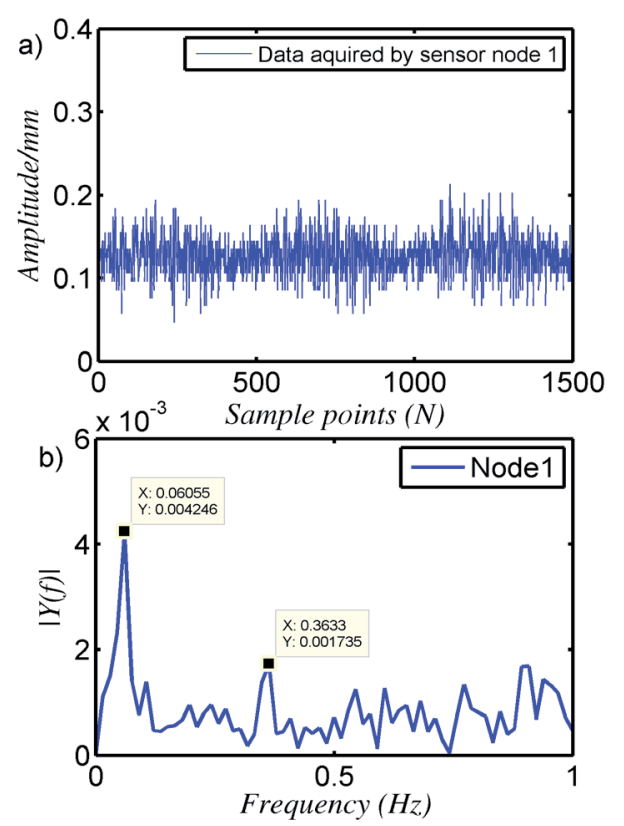

Figure 12. The measurement result of the node1

\section{CONCLUSIONS}

A distributed data acquisition measurement system for low-speed machines based on WSN is designed in this research. The system architecture, hardware design, software implementation and system validation in libratory and in real industry field are introduced in detail. Actual measurement results showed that when the sensor nodes continuously acquired and sent data, the energy conversion efficiency was higher than $90 \%$ and the nodes' power consumption came to about $110 \mathrm{~mW}$. The network was built on MESH topology, and the data transmission was stable and the packet error rate (PER) was lower than 1\% when the data sending frequency of each sensor node was $9,600 \mathrm{bps}$. And it is able to meet the engineering requirements in industry field. The acquisition precision of nodes could reach $0.01 \mathrm{mV}$. The software was developed by adopting the multi-loop state machines based on producerconsumer queue, and it operated stably and was easily extensible. Also the WSN system could be deployed for temporary or long-time installation. Experiment results indicated that the WSN system designed met the requirements for on-site data acquisition and monitoring of lowspeed machines.

\section{REFERENCES}

[1] Rusinski, Eugeniusz, Zaklina Stamboliska, and Przemysław Moczko. "Proactive control system of condition of low-speed cement machinery." Automation in Construction 31 (2013): 313324. http://dx.doi.org/10.1016/j.autcon.2012.12.001

[2] Jamaludin, N., David Mba, and R. H. Bannister. "Condition monitoring of slow-speed rolling element bearings using stress waves." Proceedings of the Institution of Mechanical Engineers, Part E: Journal of Process Mechanical Engineering 215.4 (2001): 245-271. http://dx.doi.org/10.1243/0954408011530488

[3] Kosse, Vladis, and Andy Tan. "Development of testing facilities for verification of machine condition monitoring methods for low speed machinery."Engineering asset management. Springer London, 2006. 192-197.

[4] Holroyd, T. "Condition monitoring of very slowly rotating machinery using AE techniques." 14th International congress on Condition monitoring and Diagnostic engineering management (COMADEM'2001). 2001. http://dx.doi.org/10.1016/b978008044036-1/50004-4
[5] Gungor, Vehbi C., and Gerhard P. Hancke. Industrial wireless sensor networks: Challenges, design principles, and technical approaches. Industrial Electronics, IEEE Transactions on 56.10 (2009): 4258-4265.

[6] Tiwari, Ankit, Prasanna Ballal, and Frank L. Lewis. "Energyefficient wireless sensor network design and implementation for condition-based maintenance." ACM Transactions on Sensor Networks (TOSN) 3.1 (2007): 1.

[7] Hou, Liqun, and Neil W. Bergmann. Novel industrial wireless sensor networks for machine condition monitoring and fault diagnosis. Instrumentation and Measurement, IEEE Transactions on 61.10 (2012): 2787-2798.

[8] Lu, Bin, and Vehbi C. Gungor. Online and remote motor energy monitoring and fault diagnostics using wireless sensor networks. Industrial Electronics, IEEE Transactions on 56.11 (2009): 46514659.

[9] Hashemian, H. M. Wireless sensors for predictive maintenance of rotating equipment in research reactors. Annals of Nuclear Energy 38.2 (2011): 665-680 http://dx.doi.org/10.1016/j.anucene.2010.09 .012

[10] Cai weiwei, Tang baoping, Huang qingqing. Design of wireless sensor network node for collectign mechanical vibration signal. Journal of vibration and shock.(2013):Vol.32 No.1.

[11] Sung, Wen-Tsai, and Yao-Chi Hsu. Designing an industrial realtime measurement and monitoring system based on embedded system and ZigBee. Expert Systems with Applications 38.4 (2011): 4522-4529. http://dx.doi.org/10.1016/j.eswa.2010.09.126

[12] Salvadori, Fabiano, et al. Monitoring in industrial systems using wireless sensor network with dynamic power management. Instrumentation and Measurement, IEEE Transactions on 58.9 (2009): 3104-3111.

[13] Wei Yang, Ping Wang. Feature and application of the MSP430 serial ultra-low-power MCU. Foreign Electronic Measurement Technology 12 (2008): 017.

[14] Baronti, Paolo, et al. "Wireless sensor networks: A survey on the state of the art and the 802.15. 4 and ZigBee standards." Computer communications $30.7 \quad$ (2007): $1655-1695$. http://dx.doi.org/10.1016/j.comcom.2006.12.020

[15] Shariff, Farihah, Nasrudin Abd Rahim, and Wooi Ping Hew. "Zigbee-based data acquisition system for online monitoring of grid-connected photovoltaic system." Expert Systems with $\begin{array}{llll}\text { Applications } & 42.3 & \text { (2015): } & 1730-1742 .\end{array}$ http://dx.doi.org/10.1016/j.eswa.2014.10.007

[16] Chase, Otavio Andre, et al. "Sensory platform architecture for IN SITU monitoring the thermal comfort in rural environments-The case study at Federal Rural University of Amazonian, Brazil." $\begin{array}{llll}\text { Measurement } & 58 & \text { (2014): }\end{array}$ http://dx.doi.org/10.1016/j.measurement.2014.08.031

[17] Wang, Zhongyuan, et al. "A LabVIEW based automatic test system for sieving chips." Measurement 46.1 (2013): 402-410. http://dx.doi.org/10.1016/j.measurement.2012.07.015

\section{AUTHORS}

Kai Zheng is with the School of Mechanical and Electronic Engineering, Wuhan University of Technology, Wuhan 430070, China. E-mail: zhengkai2001@163.com.

Yun Zhang is with the School of Mechanical and Electronic Engineering Wuhan University of Technology, Wuhan 430070, China. And he is the corresponding author of this paper. E-mail: whkasco@aliyun.com.

Lei Liu is with the School of Mechanical and Electronic Engineering, Wuhan University of Technology, Wuhan 430070, China.

Chen Zhao is with the School of Mechanical and Electrical Engineering, Wuhan University of Technology, Wuhan 430070, China.

This research is supported by Hubei Digital Manufacturing Key Laboratory, China. Submitted 07 September 2015. Published as resubmitted by the authors 30 December 2015 . 